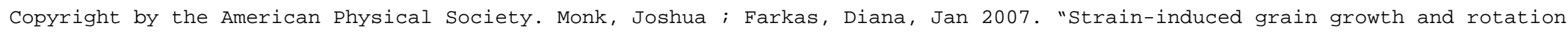
in nickel nanowires," PHYSICAL REVIEW B 75(4): 045414. DOI: 10.1103/PhysRevB.75.045414.

PHYSICAL REVIEW B 75, 045414 (2007)

\title{
Strain-induced grain growth and rotation in nickel nanowires
}

\author{
Joshua Monk and Diana Farkas \\ Department of Materials Science and Engineering, Virginia Tech, Blacksburg, Virginia 24061, USA
}

(Received 7 September 2006; published 10 January 2007)

\begin{abstract}
Virtual tensile tests of nanocrystalline nickel wires of initial $5 \mathrm{~nm}$ grain size were simulated at strain rates varying from $3 \times 10^{7} \mathrm{~s}^{-1}$ to $1 \times 10^{9} \mathrm{~s}^{-1}$ at $300 \mathrm{~K}$, reaching deformation levels up to $36 \%$. The virtual tensile tests allowed the study of the strain rate sensitivity of these nanowires, yielding an activation volume of $\sim 2 b^{3}$, where $b$ is the Burger's vector, consistent with grain boundary mechanisms of plasticity. Most importantly, after $3 \%$ deformation the grain size increased significantly during the deformation, with larger grains growing at the expense of the smaller ones as the deformation levels increase. The volume of each grain was monitored as a function of time and stress level. The results clearly indicate that the observed grain growth is stress driven, with grain size versus stress behavior being only weakly dependent on the strain rate and simulation time. Grain growth is also accompanied by grain rotation. The observations are interpreted in terms of the coupling of the relative motion of the grains parallel to the boundary and the motion of the boundary in the direction perpendicular to itself. Our results are consistent with a model where the deformation is accommodated by grain boundary sliding which in turn is coupled with grain boundary migration and rotation, producing grain growth.
\end{abstract}

DOI: $10.1103 /$ PhysRevB.75.045414

PACS number(s): 62.20.Fe, 31.15.Qg, 61.72.Mm

\section{INTRODUCTION}

The properties of nanocrystalline metals have been a focus for computational materials scientists in recent years. ${ }^{1-8}$ The capabilities of massively parallel computers have allowed large scale atomic level simulations of deformation behavior of materials with features at the nanoscale. Simulation work is critical in understanding the underlying mechanisms of plastic deformation at different strain rates, grain sizes, and structures. Molecular dynamics has been utilized in this kind of simulation, with the limitation of the small sizes and extremely fast strain rates that are imposed by the computational resources. Nanocrystalline structures are of particular interest because of their increased hardness and because of the appearance of particular phenomena at the nanoscale such as the reversal of the Hall-Petch effect. ${ }^{1}$

One particular observation in the deformation behavior of nanocrystalline materials has been the fact that nanograins are experimentally seen to grow as they are deformed. Several investigators have found experimental evidence of significant grain growth and rotation at room temperature for these small grain sizes at high deformations. ${ }^{9,12}$ In the experimental studies, it is difficult to determine the role of thermally activated processes in the observed grain growth, since there may be questions regarding the actual local temperature of the sample and whether there is actually enough time for thermally activated growth to occur. Stress enhanced grain boundary mobility was also observed in simulations. ${ }^{8,13}$

In this work, we report simulation studies of the deformation of polycrystalline Ni nanowires carried out at different strain rates and controlled room temperature. Under these conditions we can study the grain growth process by monitoring the evolution of the volume of individual grains. This process, repeated for various strain rates allows us to separate stress driven growth from any time-dependent contribution. Analysis of the simulation results show that the growth process is fundamentally driven by the stress.

\section{SIMULATION TECHNIQUE}

The virtual tensile tests simulations were performed using a conventional molecular dynamics (MD) algorithm to rescale the coordinates along the $z$ direction. The interaction between the atoms is modeled using an embedded-atom method (EAM) potential developed by Voter and Chen for nickel. ${ }^{14}$

The sample was prepared in two steps. Initially a cubic sample of $22 \mathrm{~nm}$ side was prepared using a Voronoi construction with an average grain size of $8 \mathrm{~nm}$.,6 A cylindrical shape with dimensions of $22 \mathrm{~nm}$ long and a five $\mathrm{nm}$ radius was then cut from the initial cube. The initial nickel cube had a grain size of $\sim 8 \mathrm{~nm}$, but the five nanometer radius cut from the cube resulted in a 170000 atom sample with $\sim 5 \mathrm{~nm}$ average grain size. To allow a meaningful comparison of the results obtained at different strain rates, all mechanical tests were performed using the same virtual nanowire sample, with the same grain structure.

The code used to run the MD tensile tests is Lammps and was developed by Plimpton. ${ }^{15}$ The technique uses a NoseHoover thermostat and barostat to control the temperature and pressure. The sample was first equilibrated for $450 \mathrm{ps}$ with periodicity along the wire axis and free surfaces parallel to the wire axis, with the temperature set at $300 \mathrm{~K}$. After the initial relaxation, the periodicity along the $z$ axis was increased at various rates to simulate an increasingly strained wire. The value of the stress in the direction of the wire axis was monitored to obtain the stress-strain behavior at various strain rates. As the deformation proceeds, snapshots of the sample were created for visualization.

\section{RESULTS}

Figure 1 shows the nanowires after 0, 90, and $270 \mathrm{ps,}$ deformation $(0,12$, and $36 \%$ strain, respectively) for a strain 

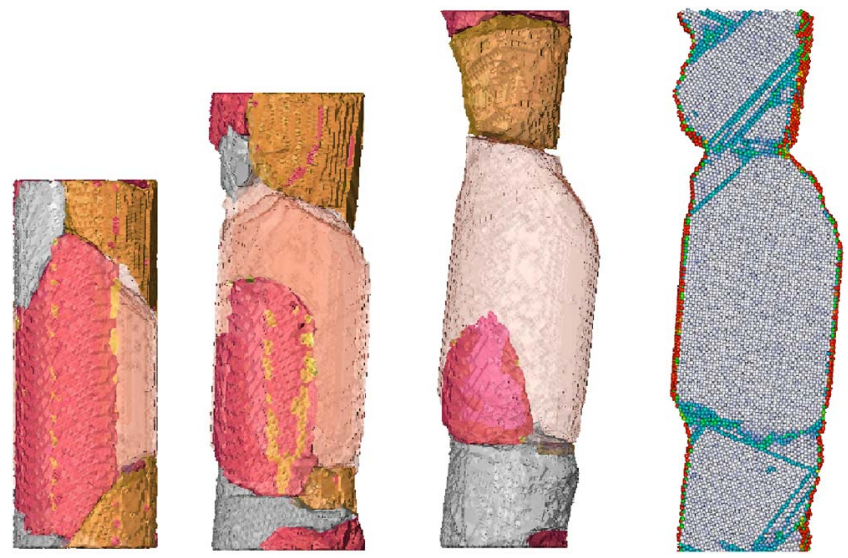

FIG. 1. (Color online) Virtual tensile test of a multigrained nickel nanowire. Snapshots at 0,12 , and $36 \%$ strain, colored according to grains. The strain rate is $1.33 \times 10^{9} \mathrm{~s}^{-1}$. The figure at right shows a slice through the wire deformed $36 \%$ colored according to the centrosymmetry parameter, visualizing stacking faults and twins in the deformed structure.

rate of $1.33 \times 10^{9} \mathrm{~s}^{-1}$. The various grains are color coded, showing significant grain growth, as the deformation proceeds. For the largest deformation, the figure also shows a slice through the center of the wire color coded according to the centrosymmetry parameter, ${ }^{16}$ visualizing stacking faults and twins in the deformed structure. Very few of these defects are seen, even at the large strain of $36 \%$, indicating that the main deformation mechanisms are grain boundary mediated. Most importantly, significant grain boundary motion is clearly seen, as the deformation proceeds. The corresponding stress strain curve is shown in Fig. 2, together with the results of tests at various other strain rates. Figure 2 shows the strain stress curves up to $5 \%$ deformation, a region that includes plastic deformation but not the higher deformation levels where the sample develops necking.

The stress strain curves show flow after deformation of about 3\%, with the flow stress decreasing for slower strain rates. To quantify the dependence of the flow stress on the strain rate, the strain rate sensitivity exponent was calculated by plotting the average flow stress over the strains of $3 \%$ to $5 \%$ as a function of the strain rate. The results are shown in Fig. 3 giving the strain rate sensitivity exponent of 0.121 for

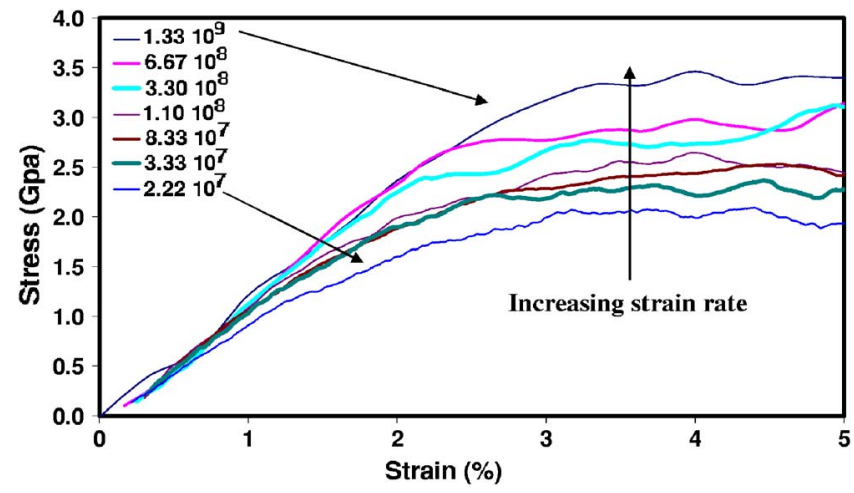

FIG. 2. (Color online) Stress strain curves for varying strain rates from $1.33 \times 10^{9} \mathrm{~s}^{-1}$ to $2.22 \times 10^{7} \mathrm{~s}^{-1}$ at $300 \mathrm{~K}$.

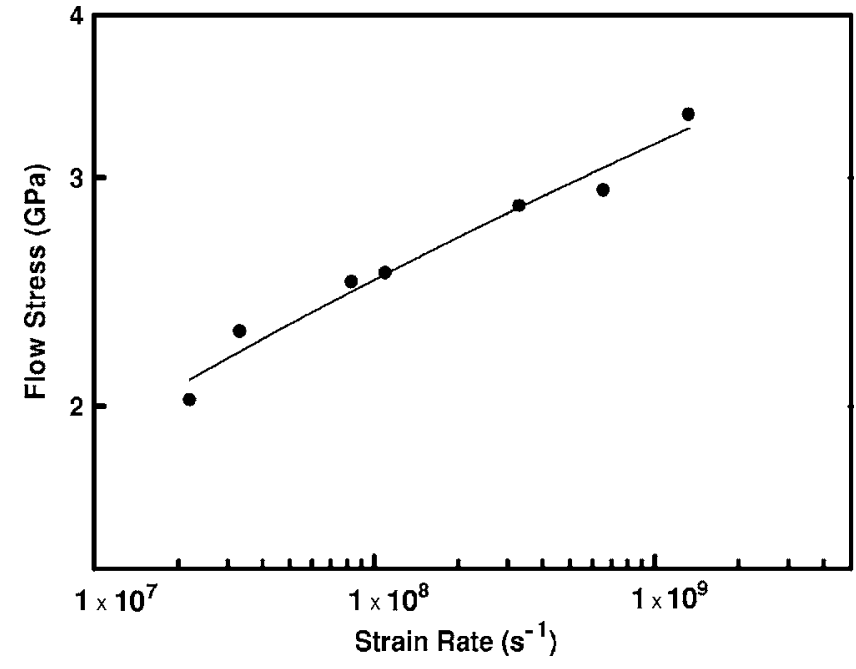

FIG. 3. The flow stress, defined from 3\% to 5\% strain, for strain rates varying from $2.22 \times 10^{7} \mathrm{~s}^{-1}$ to $1.33 \times 10^{9} \mathrm{~s}^{-1}$. Both scales are logarithmic.

the $\sim 5 \mathrm{~nm}$ grain sized nanowire. The activation volume for the deformation process ${ }^{17-20}$ can be calculated from this exponent, as follows:

$$
v^{*}=\frac{\sqrt{3} k T}{n \sigma},
$$

where $n$ is the stress sensitivity exponent, $k$ is Boltzmann's constant, $T$ is the temperature, and $\sigma$ is the flow stress. Using a flow stress value of $2.0 \mathrm{GPa}$ and the temperature of $300 \mathrm{~K}$ we obtain $0.029 \mathrm{~nm}^{3}$ or $1.878 b^{3}$, where $b$ is the Burger's vector value of $0.25 \mathrm{~nm} \cdot{ }^{13,15}$ A value of $1-2 b^{3}$ for a grain size of $\sim 5 \mathrm{~nm}$ suggests that the deformation mechanisms involves only a few atoms motion at a time, as expected in grain boundary accommodation mechanisms. Indeed, grain boundary mechanisms of plasticity have been postulated for grain sizes smaller than $20 \mathrm{~nm}$, based on extensive simulation work. ${ }^{1-6}$ In recent experimental work ${ }^{17-20}$ the apparent activation volume was found to be $20 b^{3}$ for nickel with a grain size of $30 \mathrm{~nm}$, indicating dislocation mediated plasticity. Our result for the activation volume is consistent with the expected relation between activation volumes and grain sizes. ${ }^{17-20}$

For additional confirmation of a grain boundary sliding mechanism operating, we studied the detailed atomistic structure of the deformed samples to observe dislocations at a strain rate of $1.33 \times 10^{9} \mathrm{~s}^{-1}$. It was found that the number of dislocations emitted was small, less than 15 total dislocations at $15 \%$ strain confirming that localized accommodation in the grain boundaries is the main mechanism of plasticity at these small grain sizes.

To study the observed grain growth process, we measured the volumes of the individual grains as the deformation proceeded at different strain rates. As an example, Fig. 4 shows grain size versus time for four representative grains deformed at a strain rate of $1.33 \times 10^{9} \mathrm{~s}^{-1}$. In this and subsequent figures the cubic root of the volume is used as a measure of grain size. The larger grains are seen to grow at the 


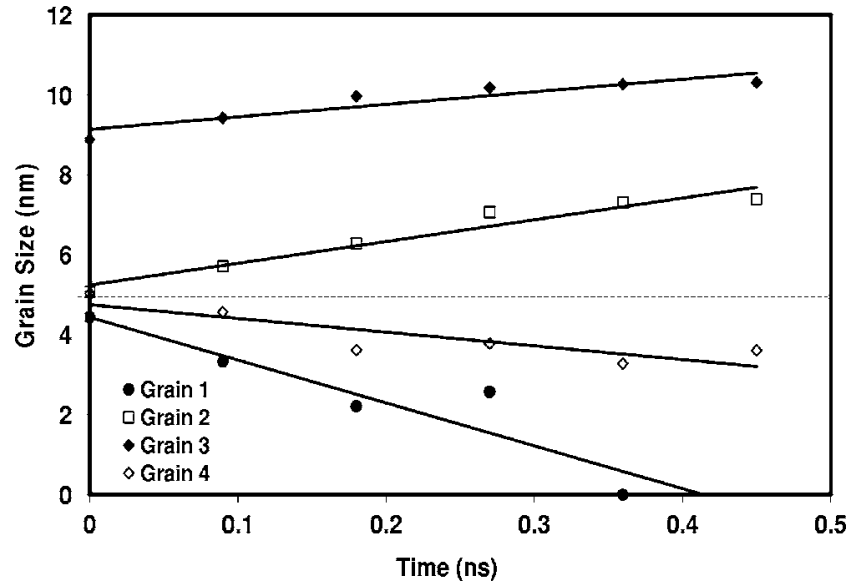

FIG. 4. Size of four individual grains, followed as a function of time for a strain rate of $1.33 \times 10^{9} \mathrm{~s}^{-1}$. The dotted line is the initial average grain size and the sizes indicated are measured as the cubic root of the grain volume.

expense of the smaller ones, a trend that was also observed for other strain rates. The initially larger grains continue to grow at the expense of smaller grains which reduce and often disappear. ${ }^{12}$ As a result of grain growth the average grain size for all grains grew as a function of time for each of the strain rates. This is shown in Fig. 5(a), which shows the
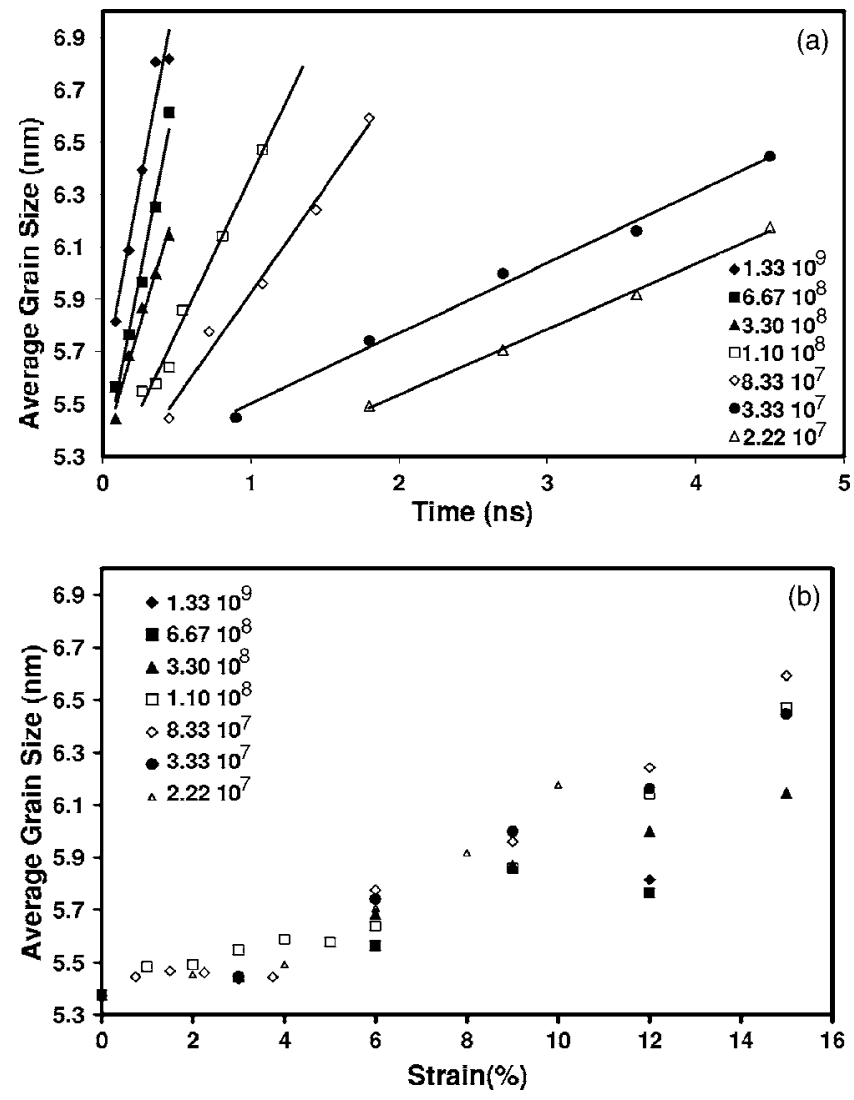

FIG. 5. (a) Average grain size as a function of time for different strain rates. (b) Average grain size as a function of strain for different strain rates. Average grain sizes are measured as the cubic root of the average grain volume and the strain rates are indicated in $s^{-1}$.

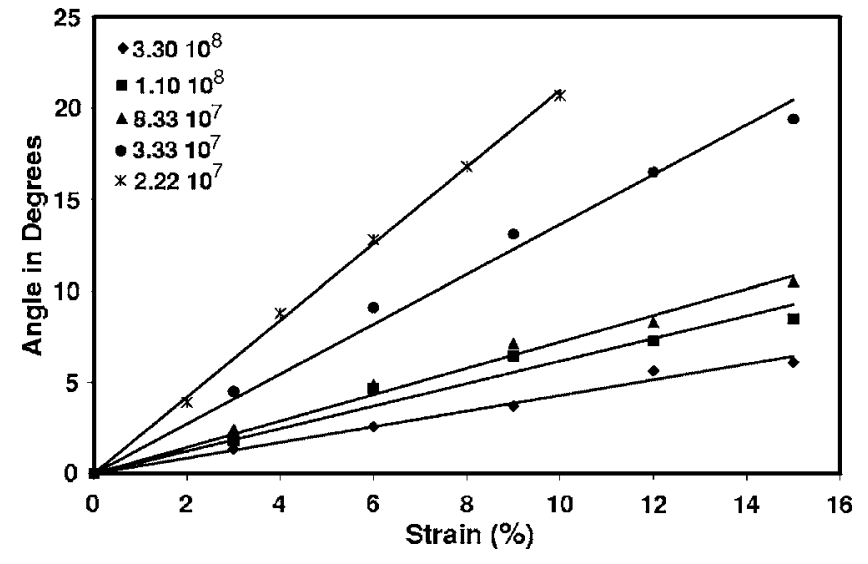

FIG. 6. Rotation angle of a sample grain in the center of the wire as a function of time for different strain rates, indicated in $s^{-1}$.

average grain size, linearly increasing over time for each strain rate. The growth rate observed for the different strain rates is very different, indicating that the growth process is controlled by the strain level, more than the time of the simulation. Figure 5(b) shows the data obtained for various strain rates, plotted as a function of strain. This figure also shows that the growth process is strongly correlated with the overall strain of the sample. The comparison of the plots of grain growth as a function of time and strain suggests that the growth process is not a thermally activated process, but rather an integral part of the mechanism of grain boundary plasticity in nanocrystalline materials. In this way the present simulations help address the controversy surrounding some experimental observations. ${ }^{10}$

Although grain growth is well recorded for increasing temperature, only recently has grain growth been experimentally observed at room temperature and postulated as due to strain. ${ }^{9-13}$ In simulation work, Schiotz et al. ${ }^{13}$ used a $5 \mathrm{~nm}$ diameter copper sample with a $10 \%$ to $-10 \%$ strained cyclic deformation and observed grain coarsening that was induced by strain. That work was performed under cyclic loading as opposed to tensile conditions. The present results, obtained at various strain rates clearly show that grain coarsening operates as part of the basic plasticity mechanism in nanocrystalline materials.

We have also analyzed the virtual tensile test for evidence of grain rotation and have found significant grain rotation during the deformation process. Grain rotation for the central grain in the wire was quantified for the various strain rates tested. Figure 6 shows the values observed for the rotation for this particular grain in the plane perpendicular to the tensile axis as a function of time. The data show that the grain rotation speed varies significantly with strain rate, indicating again that it is not a time controlled thermal process and is mostly driven by the strain itself.

\section{DISCUSSION}

Our simulations show that grain boundary motion and rotation are present as basic deformation mechanisms in these 


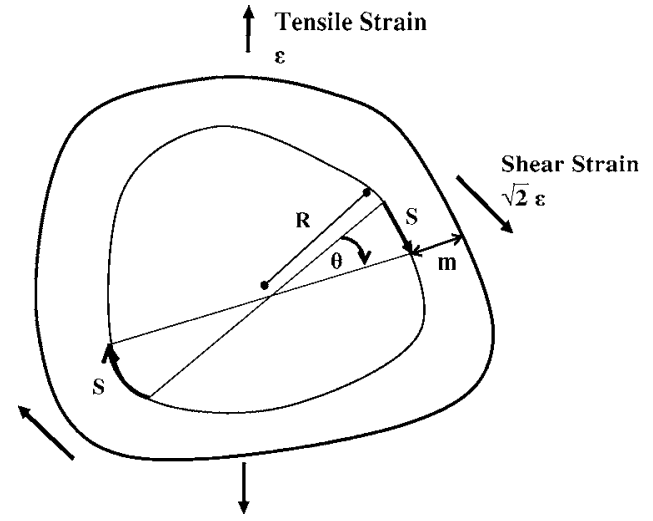

FIG. 7. Schematics of coupled sliding, migration, and rotation for an average grain in the sample.

nanowires, as have been observed in several experimental studies of the mechanical response of nanocrystalline metallic materials. The most likely mechanism responsible for the strain-driven grain growth in our simulations arises from the GB sliding that accommodates the strain. The present results can be interpreted as due to the coupling between the parallel and perpendicular motions of the grain boundary, as described by Bishop et al. ${ }^{21}$ More recently, Hyde, Farkas, and Caturla $^{22}$ analyzed the motion of a particular $\Sigma=5$ grain boundary subject to shear in $\mathrm{Fe}$ and found that the boundary moved through the nucleation and motion of displacement shift complete (DSC) lattice dislocations. This produced not only relative motion of the grains parallel to the boundary but also motion of the boundary in the direction perpendicular to itself, as a result of the same unique process. Cahn, Mishin, and Suzuki ${ }^{23}$ analyzed the atomic mechanisms of grain boundary motion for a large number of boundaries and similarly found a coupling of the motion parallel and perpendicular to the boundary when the boundary is subject to stress. In that work, they analyzed a series of boundaries and studied the values for the coupling coefficient $\beta=s / m$ where $s$ is the relative movement of the grains parallel to the boundary (sliding) and $m$ is the movement of the boundary in the direction perpendicular to itself (migration). They found values for $\beta$ that range from 0.3 to 1.5 .

For the interpretation of the present results, a simple model can be constructed assuming that:

(i) A tensile strain $\varepsilon$ is the result of a shear strain of $\varepsilon / \cos \left(45^{\circ}\right)$ for each grain.

(ii) The deformation is accommodated by sliding of the boundaries, denoted by $S$.

(iii) This movement $S$ produces a coupled migration of the boundary denoted by $m$.

(iv) Sliding in opposite directions for opposite sides of a grain also results in coupled grain rotation.

Using these assumptions (Fig. 7) the average sliding for each boundary can be calculated as the average grain radius times the average shear deformation of each grain or:

$$
S=R \sqrt{2} \varepsilon,
$$

where $R$ is the average radius of a grain. Using this equation we obtain an average displacement of the boundary parallel to itself of $(4.2 \varepsilon)$ in $\mathrm{nm}$. Analysis of the data in Fig. 5(b) yields an average grain growth of $(6 \varepsilon)$ in nm, or an average movement of the boundary perpendicular to itself $m$ of half that value, $(3 \varepsilon)$ in $\mathrm{nm}$. This means that our results imply a coupling factor $\beta=s / m$ of 1.3 consistent with the values reported by Cahn, Mishin, and Suzuki. ${ }^{23}$ In addition, if opposite sides of a grain slide in different directions, a net grain rotation will result which we can estimate in radians as

$$
\theta=S / R
$$

Substitution of $S$ from Eq. (2) gives a grain rotation of $1.41 \varepsilon$ in radians, or about $12^{\circ}$ for a $15 \%$ deformation. This value can be compared to our observed values for $15 \%$ deformation which range from 7 to $18^{\circ}$.

Therefore, the present results for grain growth can be consistently interpreted as resulting from the coupled motion of the grain boundaries. The observed grain rotation is also consistent with a picture where deformation occurs through grain boundary sliding, occurring through the motion of grain boundary dislocations, which in turn results in grain rotation and grain growth. Figure 7 shows a schematic of the process, depicted for an average growing grain. This process can have general validity as long as the grain boundary migration of each grain satisfies compatibility conditions. The compatibility conditions can be easily satisfied for small nanowire configurations such as the one studied here but can impose additional constrains in a different configuration. In addition, the effects of the free surfaces in the nanowire can be significant. For example, Derlet and Van Swygenhoven ${ }^{24}$ have shown that the presence of the free surface increases grain boundary sliding. One should also bear in mind that the present results were obtained for only one nanowire diameter and grain structure. These effects may change the magnitude of the observed grain growth but the basic nature of the coupling effects discussed here is expected to be of more general validity and possibly extend to polycrystalline materials with grain sizes in the nanometer range. Indeed, grain boundary migration driven by the strain was found in simulations performed for nanocrystalline $\mathrm{Ni}$ of $5 \mathrm{~nm}$ grain size without free surfaces. These results are reported in a recent paper, ${ }^{25}$ where significant growth of particular grains at the expense of others is shown.

\section{ACKNOWLEDGMENTS}

This work was supported by NSF, Materials Theory. We acknowledge helpful discussions with H. Van Swygenhoven and Y. Mishin. This work was performed using Virginia Tech's System X supercomputing facility. 
${ }^{1}$ J. R. Weertman, D. Farkas, K. Hemker, H. Kung, M. Mayo, R. Mitra, and H. Van Swygenhoven, MRS Bull. 24, 44 (1999).

${ }^{2}$ J. Schiotz and K. Jacobsen, Science 301, 1357 (2003).

${ }^{3}$ J. Schiotz, T. Vegge, F. Tolla, and K. Jacobsen, Phys. Rev. B 60, 11971 (1999).

${ }^{4}$ J. Schiotz, Scr. Mater. 51, 837 (2004).

${ }^{5}$ H. Van Swygenhoven, M. Spaczer, A. Caro, and D. Farkas, Phys. Rev. B 60, 22 (1999).

${ }^{6}$ H. Van Swygenhoven, A. Caro, and D. Farkas, Mater. Sci. Eng., A 309, 440 (2001).

${ }^{7}$ D. Farkas and X. Curtin, Mater. Sci. Eng., A 412, 316 (2005).

${ }^{8}$ A. J. Haslam, D. Moldovan, V. Yamakov, D. Wolf, S. R. Phillpot, and H. Gleiter, Acta Mater. 51, 2097 (2003).

${ }^{9}$ E. Ma, Science 305, 623-624 (2004).

${ }^{10}$ Z. W. Shan, E. A. Stach, J. M. K. Wiezorek, J. A. Knapp, D. M. Follstaedt, and S. X. Mao, Science 305, 654 (2004); see also M. W. Chen and X. Yan, ibid. 308, 356 (2005); Z. W. Shan, E. A. Stach, J. M. K. Wiezorek, J. A. Knapp, D. M. Follstaedt, and S. X. Mao, Science 308, 5720 (2005).

${ }^{11}$ Kai Zhang, J. R. Weertman, and J. A. Eastman, Appl. Phys. Lett. 85, 5197 (2004).

${ }^{12}$ Kai Zhang, J. R. Weertman, and J. A. Eastman, Appl. Phys. Lett. 87, 061921 (2005).
${ }^{13}$ J. Schiotz, Mater. Sci. Eng., A 375-377, 975 (2004).

${ }^{14}$ A. F Voter and S. F. Chen, Mater. Res. Soc. Symp. Proc. 82, 175 (1987).

${ }^{15}$ S. J. Plimpton, J. Comput. Phys. 117, 1 (1995).

${ }^{16}$ C. L. Kelchner, S. J. Plimpton, and J. C. Hamilton, Phys. Rev. B 58, 11085 (1998).

${ }^{17}$ R. J. Asaro and S. Suresh, Acta Mater. 53, 3369 (2005).

${ }^{18}$ L. Lu, R. Schwaiger, Z. W. Shan, M. Dao, K. Lu, and S. Suresh, Acta Mater. 53, 2169 (2005).

${ }^{19}$ Y. M. Wang and E. Ma, Mater. Sci. Eng., A 375, 46 (2004).

${ }^{20}$ Y. M. Wang, A. V. Hamza, and E. Ma, Appl. Phys. Lett. 86, 241917 (2005).

${ }^{21}$ G. H. Bishop Jr., R. Harrison, T. Kwok, and S. Yip, J. Appl. Phys. 53, 5596 (1982).

${ }^{22}$ B. Hyde, D. Farkas, and M. J. Caturla, Philos. Mag. 85, 3795 (2005).

${ }^{23}$ J. W. Cahn, Y. Mishin, and A. Suzuki, Philos. Mag. 86, 25 (2006).

${ }^{24}$ P. M. Derlet and H. Van Swygenhoven, Philos. Mag. A 82, 1 (2002).

${ }^{25}$ D. Farkas, A. Froseth, and H. Van Swygenhoven, Scr. Mater. 55, 695 (2006). 